\title{
Circuit
}

Musiques contemporaines

\section{Le laboratoire musical de l'Université Simon Fraser : une entrevue avec Barry Truax}

\section{Sylvia L’Écuyer}

Volume 19, numéro 3, 2009

Pionniers canadiens de la lutherie électronique

URI : https://id.erudit.org/iderudit/038257ar

DOI : https://doi.org/10.7202/038257ar

Aller au sommaire du numéro

Éditeur(s)

Les Presses de l'Université de Montréal

ISSN

1183-1693 (imprimé)

1488-9692 (numérique)

Découvrir la revue

Citer cet article

L'Écuyer, S. (2009). Le laboratoire musical de l'Université Simon Fraser : une entrevue avec Barry Truax. Circuit, 19(3), 48-57.

https://doi.org/10.7202/038257ar

\section{Résumé de l'article}

Le Sonic Research Studio de l'Université Simon Fraser en Colombie-Britanique a été fondé en 1965 par R. Murray Schafer. Le professeur Barry Truax y est entré en 1973 pour travailler au World Soundscape Project. Il a par la suite participé à toutes les étapes de son développement et a même dessiné le studio actuel en collaboration avec Dave Murphy. Dans une conversation avec Sylvia L'Écuyer, il décrit l'approche unique adoptée par le studio à l'intérieur de la School for Contemporary Arts, une approche multidisciplinaire et mcluhanesque. Formé à McGill, Utrecht et Stockholm, Barry Truax met en perspective les différentes philosophies et les technologies adoptées en Europe et dans l'Est du continent nord-américain. Le studio de Simon Fraser d'aujourd'hui, muni d'un équipement analogique et numérique, riche des archives qui y sont pieusement conservées, garde pour lui le meilleur des deux mondes et joue un rôle essentiel de mentorat et de socialisation auprès des étudiants. 


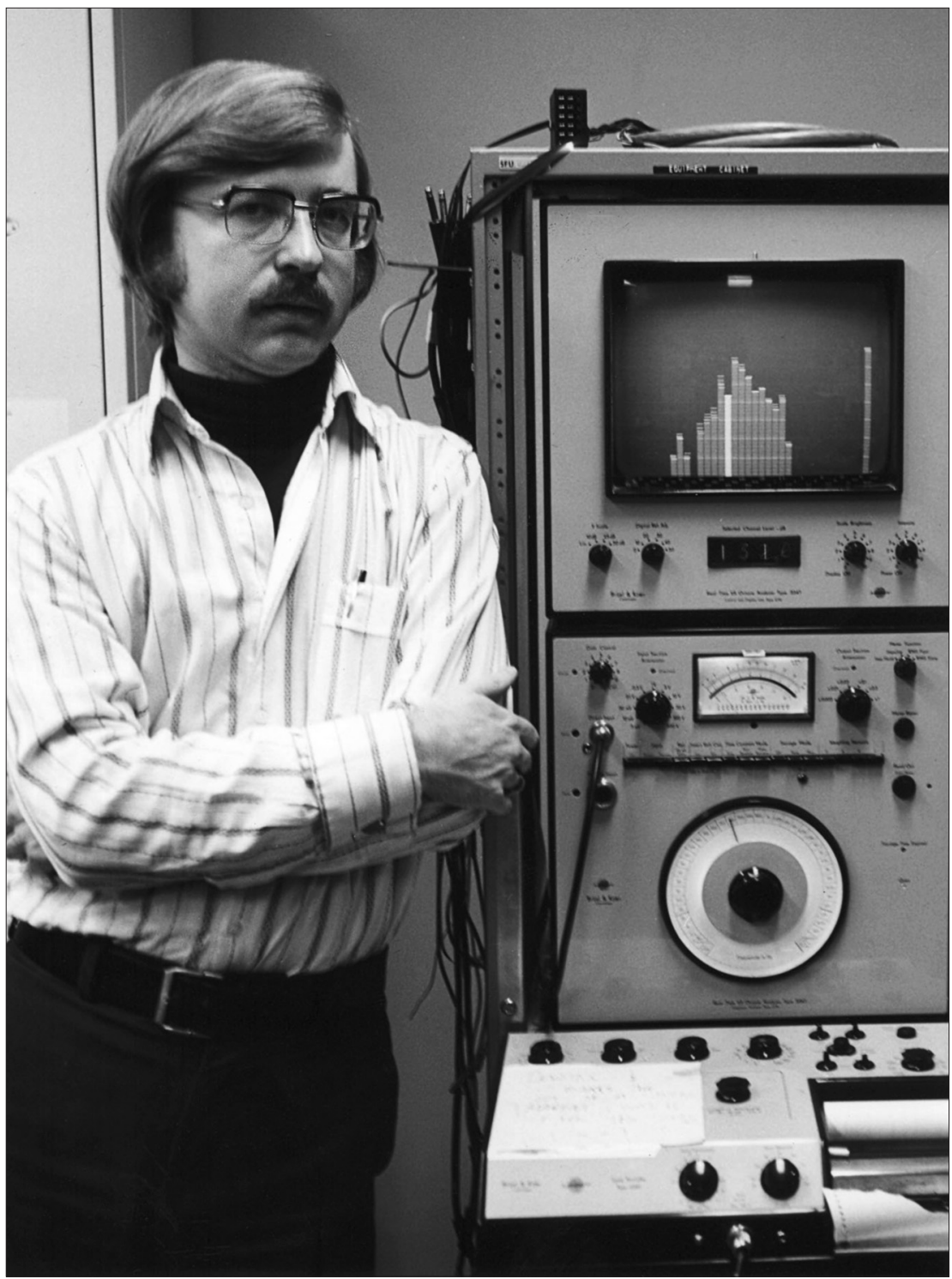

Barry Truax dans le Sonic Research Studio de I'Université Simon Fraser, 1977. (C) Barry Truax, reproduit avec autorisation. 


\section{Le laboratoire musical de l'Université Simon Fraser: une entrevue avec Barry Truax ${ }^{1}$}

Sylvia L'Écuyer

L'histoire disparaît et la technologie évolue si rapidement que je suis devenu le représentant d'une époque révolue. (BT)

SL: Quand et où a été installé le premier studio de musique électroacoustique sur la côte ouest canadienne?

BT: Deux studios ont démarré simultanément en 1965 à l'Université de la Colombie-Britannique (UBC) et à l'Université Simon Fraser $(\mathrm{SFU})^{2}$. Celui de Cortland Hulberg à l'UBC était déjà bien installé quand j’y suis arrivé en 1969. J'étais un réfugié du programme de physique et de mathématiques de l'Université Queens où la musique était presque totalement absente. Mon histoire est typique de celle des jeunes hommes de ma génération: les meilleurs élèves étaient dirigés vers les sciences, même si leur intérêt était plutôt tourné vers la musique et les arts. J'étais un pianiste assez médiocre, mais, piqué par le démon de la composition, je faisais le désespoir de mon professeur d'harmonie. Quand j'ai appris l'existence d'un studio électroacoustique à l'Université McGill, je suis allé rencontrer István Anhalt pour une entrevue. J'étais terrifié devant la figure imposante de cet homme qui représentait pour moi la culture européenne, mais nous sommes devenus de bons amis par la suite. Anhalt avait fondé le studio de McGill en 1962, après avoir été formé à l'Université de Toronto dans ce qui a été le premier studio au Canada, créé par Arnold Walter au milieu des années 1950 et qui a été équipé des instruments développés par Hugh Le Caine du Conseil national de recherches?
1. Entretien réalisé le $1^{\text {er }}$ avril 2009 à Vancouver.

2. À l'occasion du $40^{e}$ anniversaire du studio en 2005, Barry Truax a produit un CD où l'on trouve des pièces de sept compositeurs qui ont travaillé au studio: Hildegard Westerkamp, John Oswald, Jean Piché, Barry Truax, Anne Holmes, Arne Engelfeldt, Martin Gotfrit. SFU 40, Cambridge Records (CSR CD 0501).

3. L'histoire du studio de Toronto est bien documentée dans l'ouvrage de Gayle Young, Blues pour saqueboute, dont des extraits sont reproduits dans ces pages. Voir également Myron Schaeffer, "The Electronic Studio of the University of Toronto", The Journal of Music Theory, vol. 7, no 1 (1963), p. 73-81. 
4. Adams, Stephen, R. Murray Schafer, Toronto, Buffalo, University of Toronto Press, coll. Canadian Composers, n ${ }^{\circ} 4$, 1983, p. xii.

5. Barry Truax a publié deux articles sur le projet Soundcape: "Soundscape studies: an introduction to the world soundscape project", Numus West, vol. 5, 1974, p. 36-39, et "Soundscape, Acoustic Communication and Environmental Sound Composition", Contemporary Music Review, vol. 15, $\mathrm{n}^{\text {os }} 1-2,1996$, p. 49-65
Sur la côte ouest, la situation était fort différente. Au lieu des instruments de Le Caine, on s'est intéressé aux synthétiseurs analogiques qui ont fait leur apparition aux États-Unis dans les années 1960, le MOOG et le BUCHLA, représentant chacun deux philosophies différentes. Tandis qu'à Berkeley, Don Buchla créait des séquenceurs, Robert Moog, sur la côte Est, mettait au point des claviers qui ont été rendus célèbres par les compositions de Wendy Carlos. Il ne faut donc pas s'étonner que les deux studios de la ColombieBritannique aient choisi de s'équiper de synthétiseurs analogiques et de séquenceurs conçus par les deux inventeurs américains, ni qu’on choisisse d'y produire de la musique électronique.

On était encore à l'époque pré-paysage sonore. Mais voilà que R. Murray Schafer, connu pour ses écrits sur l'éducation et la créativité, est arrivé à Vancouver, attiré par l'ouverture d'une toute nouvelle université. Moderne, mcluhanesque dans sa conception, avec un curriculum ouvert et une approche essentiellement expérimentale, la SFU s'était immédiatement dotée d'un studio de musique électroacoustique. Dans la biographie de R. Murray Schafer ${ }^{4}$, on peut voir une photo du studio d'origine et l'équipement qui s'y trouve est pratiquement le même que celui que nous avons utilisé jusque dans les années 1980. Célèbre pour le fameux World Soundscape Project (WSP), élaboré entre 1969 et 19715, Schafer a acquis une réputation de compositeur de musique électronique, mais en fait il n'en a pas beaucoup réalisé lui-même. Il y a bien un accompagnement sur bande pour Music for the Morning of the World, ces merveilleuses mélodies écrites pour Phyllis Mailing, et aussi pour quelques autres de ses œuvres, mais c'est tout. En 1971, Schafer entre au tout nouveau «Department of Communications Studies» de l'université, qui va accueillir le studio. Il y amène des étudiants, commence à y enseigner la théorie du Soundscape Project et, grâce, à une subvention de l'UNESCO, achète un magnétophone à bande magnétique NAGRA et part sur le terrain. Les premiers enregistrements ont été réalisés à Vancouver en 1972. Nous avons toujours ces rubans; ils sont conservés dans la médiathèque du Sonic Research Studio.

C'est au Département des communications, une entité interdisciplinaire, que le concept d' "environnement sonore » et le projet « Soundscape » (paysage sonore) ont trouvé leur vraie dimension. D'abord essentiellement artistique, le projet a peu à peu glissé vers une perspective de sciences sociales. Bien sûr, Schafer s'était entouré de jeunes compositeurs. Quand je suis entré au studio, Hildegard Westerkamp s'y trouvait déjà. Comme moi, elle avait assisté à une conférence de Schafer à l'UBC et elle avait été fascinée. Pour ma part, je suis allé le rencontrer, il a accepté de signer une lettre de 
recommandation adressée au Conseil des Arts pour ma demande de perfectionnement en Europe et je suis parti pour deux ans. C'est seulement à mon retour en 1973 que j’ai pris connaissance du World Soundscape Project.

SL: C'était donc tout à fait nouveau ce projet ou avez-vous rencontré des initiatives similaires pendant votre séjour en Europe de 1971 à 1973?

BT: Non, c'était unique et le projet s'est cristallisé sous l'impact de divers facteurs dans le climat très favorable de la côte Ouest. L'influence de Marshall McLuhan, le mouvement écologique, l'activisme environnemental de la Fondation Greenpeace, qui était basée ici même à Vancouver, et jusqu'à la configuration du campus ont été des facteurs déterminants.

La SFU était une université toute neuve, qui se distançait des traditions de l'UBC en se fondant sur l'interdisciplinarité, avec un concept architectural unique et une proximité incroyable avec la nature. Le sommet sur lequel le campus est construit est entouré de montagnes et, à l'époque de sa construction, le débat était engagé sur la conservation de l'environnement aux portes d'une ville moderne en pleine croissance. Murray Schafer habitait à North Vancouver et le calme de sa résidence au bord de l'eau était sans cesse troublé par le bruit des hydravions qu'il abhorrait. La lutte contre la pollution sonore l'avait déjà mobilisé. Il ne faut pas oublier non plus que Schafer, avant d'être compositeur, était connu comme un humaniste et un éducateur. Il avait publié de la correspondance du XIXe siècle, des études sur Ezra Pound ${ }^{6}$ et E. T. A. Hoffmann7. Quand on lit The Tuning of the World ${ }^{8}$, le livre qu'il a conçu à partir de son article «Music of the environment» publié à Vienne en 1973, on voit que toutes ces influences se rejoignent et l'on reconnaît en lui un chercheur du secteur des humanités plutôt qu'un compositeur. Quand il décrit les changements survenus dans notre environnement sonore, quand il observe la façon dont nous écoutons, son approche est sociologique.

Techniquement, le projet Soundscape était solide. Schafer a pris soin d'utiliser le meilleur équipement disponible, et ces enregistrements sont toujours en excellent état encore aujourd'hui, surtout ceux qui ont été réalisés au Canada à partir de 1973 et en Europe à partir de 1975 avec un ruban d'excellente qualité, le Scotch 206/207. Nous avons presque entièrement numérisé ces bandes, à l'exception de celles qui ont été réalisées à Vancouver, et nous avons de la chance car elles n'ont rien perdu de leur qualité originale. Le catalogue des enregistrements, qui totalisent environ 300 heures, est disponible sur le site internet du studio9 et nous avons le projet de les transférer sur notre portail internet, parce que les rubans DAT sur lesquels on les conserve sont eux aussi menacés de détérioration.
6. "Ezra Pound and Music", Canadian Music Journal, vol. 5, 1961, p. 15-43; traduction partielle par Pierre Alien sous le titre "Ezra Pound et la musique", Les Cahiers de L'Herne, $n^{0} 7$, 1965.

7. E. T. A. Hoffmann and Music, Toronto, University of Toronto Press, 1975.

8. The Tuning of the World, Toronto et New York, Knopf, 1977; paru en français sous le titre Le Paysage sonore, Paris, J.C. Lattès, 1991.

9. <http://www.sfu.ca/sonic-studio/srs/ index.html $>$. 
10. Initialement appelé le STEM, puis Instituut voor Sonologie à l'Université d'Utrecht, le studio a été fondé en 1960 - parmi ses principaux collaborateurs, on trouve Gottfried Michael Koenig, Konrad Boehmer, Frits Weiland, Dick Raaijmakers, Ton Bruynel et Rainer Riehn. Voir le site <http://sonhors.free.fr/ kronik/sonology.htm>.

11. On peut voir des photos sur le site de Barry Truax à l'adresse suivante: <http:// www.sfu.ca/ truax/index.html>.
SL: Vous avez tout de même été en contact avec Murray Schafer pendant votre séjour d'études en Europe.

BT: Oui, mais j'ai aussi découvert de nouvelles technologies. Je me suis d'abord inscrit à l'Institut de sonologie d'Utrechtt ${ }^{10}$ où Gottfried Koenig venait tout juste d'acheter un ordinateur PDP15 ${ }^{11}$. Pendant ces deux années, je me suis également rendu à l'Elektron Music Studio de Stockholm. En ce lieu se trouvait le «chaînon manquant» si je puis dire, l'ordinateur personnel qui permettait de piloter des instruments électroniques. Ces ordinateurs «personnels » ont l'air énormes à nos yeux d'aujourd'hui, mais à l'époque ils représentaient déjà une avancée immense : je n'ai jamais eu à travailler avec des cartes perforées et des traitements par lots. C'était un procédé interactif en temps réel, incroyablement limité c'est sûr, avec un convertisseur à 12 bits et une seule voix monophonique. Mais c'était déjà une nouvelle façon de composer, alliant la précision de la synthèse numérique et l'interaction, à partir d'un clavier, sans capacité graphique. J'ai vraiment le sentiment d'avoir été au bon endroit au bon moment pour être initié à la programmation; l'on pouvait y passer des heures, parfois des nuits! En 1973, John Chowning de l'Université Stanford est venu à Utrecht. Il nous a fait découvrir la synthèse FM, la modulation de fréquence, et j’ai pu réaliser ma première synthèse en temps réel.

Utrecht était un laboratoire de pointe: contrôle par ordinateur, mixage quatre pistes, synthèse numérique élémentaire et composition interactive. Il faut bien se rendre compte qu'à l'époque, personne en dehors de l'Institut, à

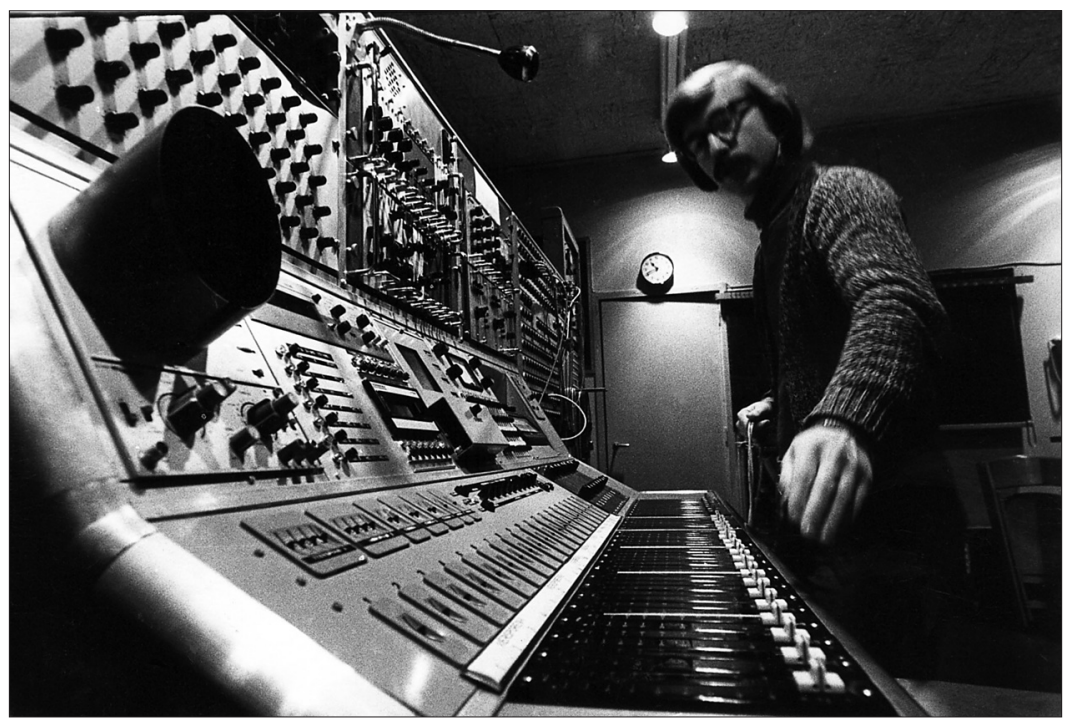

Barry Truax à l'Institut de Sonologie d'Utrecht, 1973.

Photo Theo Coolsma. (c) Barry Truax, reproduit avec autorisation. 
moins d'être un ingénieur, n'avait entendu parler des ordinateurs. On était encore loin de l'audionumérique, des ordinateurs portables; cette technologie était réservée au cercle très restreint de l'Institut. Et dès qu'on en sortait, on se trouvait au milieu d'une ville européenne aux vieilles rues étroites et bruyantes qui n'avaient jamais été conçues pour une circulation aussi intense. Alors quand Murray Schafer m'a proposé de faire des enregistrements pour le projet Soundscape à Utrecht, j'ai commencé à faire des soundwalks (promenades sonores) et à enregistrer des échantillons en vue de me préparer à aller travailler avec lui à SFU. C'était comme prendre une bouffée d'air frais. On avait l'impression d'être à l'avant-garde dans le climat de vase clos de l'Institut. À l'extérieur, le bruit était effroyable, avec tous ces bus et ces camions qui s'engouffraient dans les rues étroites. Heureusement, il y avait aussi les rues riveraines des canaux, la zone piétonnière de l'Oudegracht par exemple où l'on pouvait trouver un peu de paix et de silence et concevoir une soundwalk. C'est à ce moment-là que tout s'est cristallisé pour moi : la musique électronique produite analogiquement, l'approche par le biais des sciences sociales, la conscience de l'environnement, l'ordinateur. En rentrant à Vancouver, j'ai bien rapporté les logiciels avec moi, mais le laboratoire ne possédait pas d'ordinateur et j'ai dû me réfugier les soirs et les week-ends au Département de psychologie ou d'informatique. Et chaque fois que le département renouvelait son équipement, je devais codifier à nouveau tout le programme. Je l'ai refait au moins trois fois. Finalement, quand l'unité qui

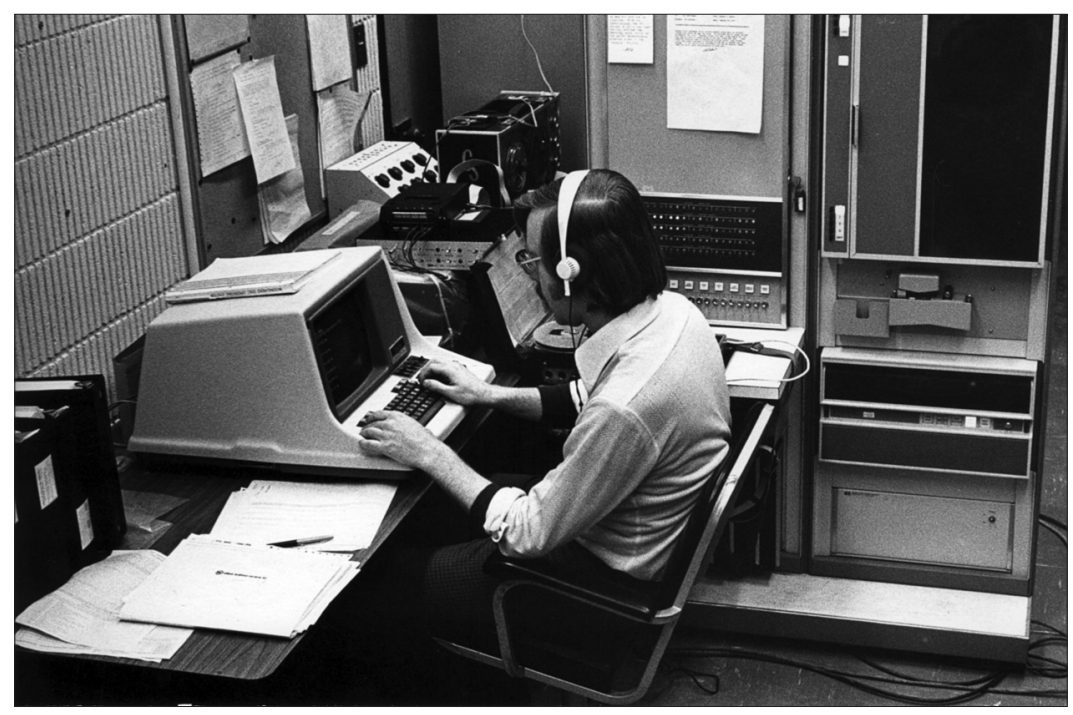

Barry Truax utilisant le système Hewlett Packard de l'Université Simon Fraser, 1978.

(C) Barry Truax, reproduit avec autorisation. 
12. Voir le contenu sur le site $<$ http:// www.sfu.ca/ truax/vanscape.html>. est devenue la «School for Contemporary Arts» a démarré son programme de musique vers 1980, nous avons enfin eu notre propre ordinateur. C'était un PDP-11, un ordinateur très performant pour l'interactivité. Si jamais quelqu'un écrit l'histoire des arts assistés par ordinateur (pas seulement la musique), on verra que, dans les années 1970, tout le monde utilisait des ordinateurs PDP fabriqués par la Digital Equipment Corporation (DEC), ou la version plus petite, le LSI. Malheureusement, ces appareils ont disparu, la compagnie rivale IBM ayant accaparé le marché des affaires et de la recherche. Il y a bien eu une version améliorée, le VAX, qui voulait concurrencer IBM dans les laboratoires et les bureaux, mais qui n’a jamais été produit en format individuel (Desktop PC). Et c'est dommage parce que ces machines permettaient de travailler en temps réel. Elles pouvaient être programmées pour effectuer toutes sortes d'applications courantes, comme la conversion du numérique à l'analogique et la sauvegarde de données. Mais le processus était lent, on travaillait en monophonie, et il a fallu attendre un nouveau type d'appareil, le DSP (Digital Signal Processor) pour franchir une nouvelle étape. Équipé de microprocesseurs, le DMX-10oo permettait d'effectuer en temps réel une synthèse assistée par ordinateur et, plus tard, de faire le traitement numérique du signal (Digital Signal Processing). Si je n'avais pas eu ces appareils, je n'aurais jamais pu effectuer la synthèse granulaire en 1986. Ma pièce, Riverrun a été réalisée grâce à ce processus. Ça a été une percée importante, mais il a fallu attendre encore quelques années pour développer de bons systèmes de conversion de l'analogique au numérique et pour traiter des sons environnementaux.

Ma première pièce écrite à partir du traitement de sons environnementaux, Pacific, a été réalisée en 1990. Avant cette date, pour des raisons purement techniques, il était impossible d'utiliser de longs fragments, on ne pouvait traiter que des échantillons très brefs, et des compositions de type soundscape étaient impossibles. On pourrait dire que Riverrun ou encore Models of River s'en rapprochaient mais elles étaient entièrement réalisées à partir de sons de synthèse granulaire. Tout le matériel sonore était généré en huit pistes, mais il fallait toujours les réduire à quatre jusque dans les années 1990, quand on a enfin eu accès à la conversion de l'analogique au numérique, au traitement numérique du signal (granulation des échantillons), au travail en huit pistes, etc.

On peut se rendre compte des progrès réalisés en comparant les deux éditions du Vancouver Soundscape, toutes deux disponibles sur CD ${ }^{12}$. La première a été produite en 1973 et la seconde en 1996. Quatre compositeurs ont participé au projet de 1996, deux Allemands et deux Canadiens, et on 
peut constater que la composition de paysages sonores s'est développée en format huit pistes avec spatialisation.

SL: Est-ce qu'à un certain moment, vous ne vous êtes pas détaché de l'échantillonnage pour vous intéresser à l'abstraction?

BT: Le paysage sonore n'est jamais vraiment abstrait: on utilise des «sons trouvés » [not abstract sounds but abstracted sounds] simplement enregistrés avec un minimum d'information sur leur provenance, manipulés légèrement de façon transparente, ou même sans aucune manipulation du tout, comme pour la pièce New Year's Eve in Vancouver Harbour qui a été interprétée pour nous par tous les navires qui se trouvaient dans le port. D'autres pièces ont l'air de «sons trouvés » comme les compositions portuaires qui se trouvent sur les deux CD, mais en fait elles sont montées en multipistes. Dans la réalité, vous ne trouveriez jamais une telle diversité sonore en quatre minutes. L'abstraction apparaît peut-être pour la première fois dans la séquence de l'arrivée au port (Entrance to the Harbour) en 1973 où l'on a simulé un trajet de Horseshoe Bay (Point Atkinson) jusqu'au Lion's Gate Bridge. Il a fallu condenser les sons et les mixer avec les appels des sirènes de brume et les cloches des bouées, enregistrés à part et intégrés par la suite. C'est un montage assez rudimentaire, une légère extrapolation si on considère le trajet original, mais avec des sons toujours extraits de l'environnement sonore naturel.

La plupart des compositeurs semblent avoir suivi cet exemple dans leur conception de paysages sonores. On retourne toujours aux sources, même si on fait appel au traitement numérique, comme le fait par exemple Hildegard Westerkamp avec les synthétiseurs. Les sons ne sont pas abstraits mais extraits. Ainsi, on peut toujours reconnaître l'origine de la pièce, surtout quand il s'agit de compositions de la côte Ouest, comme Talking Rain et Beneath the Forest Floor. Au début de cette dernière, par exemple, on entend des coups sourds répétés sans qu'on puisse identifier leur provenance'13. Mais c'est tellement évocateur du sous-sol de la forêt, que la source sonore n'a pas vraiment d'importance.

Je ne vais pas entrer dans le détail d'analyse d'une pièce parce que ce n'est pas notre propos ici, mais il reste que la démarche adoptée par notre studio dans la composition des paysages sonores été suivie partout: on est passés de la phonographie (les sons trouvés) aux sons extraits et manipulés, toujours en gardant le lien avec le contexte sonore original. Et c'est vraiment ce qui nous distingue fondamentalement de l'école électroacoustique européenne ou de l'école acousmatique montréalaise. Il y a bien des compositeurs montréalais, Yves Daoust par exemple, qui se sont intéressés à la musique anecdotique. Après tout, nous avons tous recours aux mêmes techniques d'échantillon-
13. On peut entendre la pièce sur le site <http://www.sfu.ca/ truax/ vanscape.html>. 
nage. Mais d'autres compositeurs, comme Francis Dhomont, qui a composé des pièces absolument magnifiques dans la tradition acousmatique, ont voulu dissocier les sons de leur source. Ils sont là pour leur pure beauté, avec d'inévitables références au monde réel (comment les éviter?), mais ce monde n'est pas le sujet de la pièce. Ce sont des œuvres d'imagination, de symbolisme, de formes, d'espaces imaginaires, tandis que le paysage sonore, c'est toujours à propos du monde réel.

SL: Vous avez un nouveau studio depuis 2006?

BT: Plus exactement, le studio a déménagé. C'est toujours le Sonic Research Studio, mais en fait toute l'École de communications (School of Communications) a déménagé dans une autre partie du campus. Les locaux sont plus ouverts (avec des fenêtres!) et il a fallu le reconfigurer, mais nous avons pris la décision de le conserver mi-analogique mi-numérique. Bien sûr, si on commençait à zéro aujourd'hui, on ne ferait jamais ça! Mais nous avons toute une histoire derrière nous, des archives qui n'ont pas été encore toutes numérisées et nous persistons à conserver un studio tandis que la plupart des institutions à petit budget aujourd'hui se contentent d'ordinateurs portables et de postes de travail individuels, ce qui est parfaitement efficace et fonctionnel. Mais un studio est aussi un lieu de rencontre et d'échange, et les étudiants aiment beaucoup se retrouver ici en petits groupes. C'est vrai que, la plupart du temps, ils travaillent seuls avec leur ordinateur portable, souvent à la maison où certains ont des équipements supérieurs à ceux que l'université peut offrir, mais le studio a une fonction de mentorat et de socialisation. Nous y travaillons ensemble, nous écoutons le travail des uns et des autres et nous échangeons nos commentaires. Évidemment, il y a en plus une fonction pédagogique qui n'est pas accessible si on s'en tient aux manuels d'instruction. Techniquement, esthétiquement, historiquement, le studio joue un rôle important.

Et puis l'équipement analogique a un avantage unique : celui de la manipulation physique en temps réel. Nous avons des étudiants qui n'ont jamais mis les mains sur une console, ce qui me semble aberrant. Je ne prêche pas un retour complet à l'analogique, mais ce qu'il y a de bien dans le système analogique, c'est l'interaction, ce que j'appelle la coordination du geste et de l'oreille. Le Sonic Research Studio aujourd'hui, tel que je l'ai conçu avec Dave Murphy qui l'a bâti, est resté en grande partie analogique, même si la plupart du travail qu'on y fait est sous forme numérique. Le concept des circuits et des consoles est analogique, mais les contrôles sont programmables et, à l'avenir, je crois que ce studio se développera en multipiste (en multiples de huit), et fera appel au traitement numérique (Digital Signal Processing). Il va aussi permettre de combiner la précision numérique avec l'interaction 
analogique. C'est le meilleur des deux mondes, parce qu'on conserve justement ce rapport du son et du geste en temps réel. C'est vrai qu'aujourd'hui on peut tout programmer, qu'on ne doit plus forcément travailler en temps réel, mais en adoptant cette pratique, on perd l'humanité du geste, la communication. On risque de passer son temps devant un écran.

SL: Est-ce que vos étudiants doivent apprendre à travailler avec l'équipement analogique et le numérique?

BT: C'est une grande question pour moi. Au début, quand le numérique n'était pas tellement développé, le travail se faisait moitié-moitié. Aujourd'hui, on n'a plus vraiment le temps de maitriser les techniques analogiques. Ce que je veux surtout, c'est montrer aux étudiants comment on avait l'habitude de travailler, c'est un peu un cours d'histoire. Il n'est pas question de leur dire qu'une méthode est supérieure à l'autre, elles ont chacune leurs forces et leurs faiblesses, mais quand ils font l'expérience de l'action en temps réel, et puisque le processus analogique est carrément physique, ils peuvent mieux comprendre ce qui se passe, mieux que de voir simplement un menu sur un écran. Il est clair que le studio analogique est condamné à disparaître : le coût et la difficulté d'entretenir et de réparer les appareils deviennent vite prohibitifs, mais en ce moment nous sommes toujours à une époque de transition et un studio historique comme le nôtre (si j'ose le qualifier ainsi, après tout il n’a que 44 ans!) est maintenant équipé principalement d'ordinateurs. J'espère qu'on conservera une interaction analogique dans un environnement numérique et que les studios d'université continueront de jouer leur rôle, qui en est essentiellement un de recherche en même temps que de production. Même au premier cycle, les étudiants font de la recherche. Sinon, on se contenterait de voir ce que le nouveau logiciel sur le marché permet de réaliser. Comme tout le monde a accès aux mêmes logiciels, il faut encourager la recherche pour trouver de nouvelles idées, accéder à de nouvelles connaissances, arriver à un résultat artistique plus intéressant. Ce n'est pas du tout évident dans un environnement où tout le monde veut avoir toujours le logiciel le plus performant.

Il y a des gens qui croient que les studios sont une chose du passé, mais je pense que nous sommes dans une position où nous avons le mandat de conserver ce qu'ils offrent de meilleur. Les étudiants arrivent aussi avec un bagage plus avancé. J'ai des élèves du secondaire qui composent déjà de la musique sur ordinateur. Après tout, ça se fait depuis vingt ans dans le monde de la musique pop et de la musique commerciale. Et ceux qui veulent explorer plus avant, eh bien ils viennent chez nous. 